\title{
Nonstructural Versus Structural Bone Grafting for Interbody Fusion in the Treatment of Lumbar Tuberculosis by a Single Posterior Approach
}

\section{Zenghui Zhao}

the First Affiliated Hospital of Chongqing Medical University

Jianxiao Li

Chongqing Emergency Medical Center

Wei Luo

the First Affiliated Hospital of Chongqing Medical University

Yong Zhu

the First Affiliated Hospital of Chongqing Medical University

Xing Du

the First Affiliated Hospital of Chongqing Medical University

Yunsheng Ou ( $\triangle$ ouyunsheng2001@163.com )

the First Affiliated Hospital of Chongqing Medical University

\section{Research Article}

Keywords: Lumbar spinal tuberculosis, Single-stage posterior debridement, interbody fusion, Nonstructural bone grafting, Structural bone grafting

Posted Date: November 1st, 2021

DOI: https://doi.org/10.21203/rs.3.rs-1023139/v1

License: (c) (1) This work is licensed under a Creative Commons Attribution 4.0 International License. Read Full License 


\section{Abstract}

Background Many types of bone grafting have been reported for successful use in the treatment of lumbar spinal tuberculosis. However, none-structural bone grafting has rarely been studied. This study was aimed to identify the clinical efficacy of nonstructural with structural bone graft for interbody fusion in the surgical treatment of single segment lumbar tuberculosis after one stage posterior debridement.

Methods We retrospectively reviewed patients with nonstructural $(n=27)$ and structural $(n=22)$ bone grafting after single-stage posterior debridement and instrumentation for lumbar tuberculosis with at least 24 months of follow-up. Plain radiographs, magnetic resonance imaging, computed tomography scans, and health-related outcomes (e.g., Visual Analog Scale for back pain, the Oswestry Disability Index) were collected and analyzed before and after surgery.

Results Both nonstructural and structural bone grafting were associated with significant improvements in the life quality parameters, the laboratory tests, and the Cobb angle of local kyphosis. A slight loss of Cobb angle correction was observed in two groups. Nonstructural bone grafting exhibited the advantages of less operation duration, blood loss and lower bone fusion rate compared with structural bone grafting. There were three complications in the nonstructural bone grafting and four complications in the structural bone grafting group, with no significant difference between two groups.

Conclusions In conclusion, nonstructural bone grafting can achieve comparable interbody fusion to structural bone grafting but has less surgical trauma and simpler surgical procedure than structural bone grafting in the treatment of single segment lumbar tuberculosis after one stage posterior debridement and pedicle screws fixation.

\section{Introduction}

Spinal tuberculous (STB) is the most common type of extra-pulmonary tuberculosis[1], and accounts for nearly $50 \%$ of skeletal tuberculosis. STB is prone to occur at thoracic and lumbar spine, leading to vertebral collapse, kyphosis and even paralysis [2-3]. The goals of spinal tuberculosis surgery are to debride lesions, decompress spinal cord and reconstruct spinal stability. Nowadays the one stage posterior surgery is widely used for the treatment of thoracolumbar TB [4-5]. Posterior fixation plays a significant role in the correction of kyphosis deformity and prevention of correction loss [6-7]. After removing the affected vertebral bone and intervertebral disc, interbody bone grafting is required to reconstruct and fuse the anterior column, although some authors vowed its unnecessity [8-10]. Many types of bone grafting have been reported for successful use, such as iliac bone, titanium mesh cage filled with bone granules, rib bone and so on[11]. To the best of our knowledge, there are few studies about nonstructural bone grafting for interbody fusion in the treatment of lumbar TB.

Our previous study demonstrated that nonstructural bone graft achieved similar curative effects as structural bone graft for single segment thoracic tuberculosis and has the advantages of less surgical trauma and shorter bone graft fusion time[12]. Lumbar spine bears more compressive load than thoracic 
spine and is devoid of the protection of rib cage. Whether nonstructural bone grafting can achieve the same clinical effect as structural bone grafting in lumbar TB? Some authors have reported the successful use of nonstructural bone graft for interbody fusion to lumbar STB[13-14], but these studies did not set control that the conclusions may be biased. Liu[15] compared the clinical efficacy of one-stage posterior debridement with nonstructural autogenous bone graft versus anterior debridement, structural bone graft combined with posterior instrumentation. The results showed the former method had a significantly shorter operation time, lower blood loss, shorter hospital stay and less hospitalization cost, except the bone graft fusion time. However, the conclusion may be biased because the surgical approaches used in the two groups were quite different.

The aim of this study was to investigate the effect of nonstructural bone grafting for interbody fusion through comparing the clinical and radiological outcomes with structural bone grafting in the single segment of lumbar TB treated by one-stage posterior debridement and instrumentation.

\section{Methods}

\section{Patients}

This study was approved by the Institutional Review Board of our hospital and the informed consent was obtained from all individual participants included in the study. The methods used in this study were in accordance with the Declaration of Helsinki. From January 2015 to July 2017, patients with the diagnosis of lumbar TB underwent one-stage posterior debridement, bone grafting and instrumentation at our spine surgery center were retrospectively analyzed. Patients with progressive neurologic deficit, persistent pain due to instability and kyphosis were the candidates for surgery.

The selection criteria were as follows: 1) Preoperative diagnosis of spinal TB is confirmed by postoperative pathological examination; 2) lumbar spinal TB with two adjacent vertebra and intervertebral disc involved; 3) The methods of bone grafting were nonstructural bone graft (autologous granular bone) or structural bone graft (titanium mesh cage filled with autologous granular bone); 4) The clinical and imaging data during the follow-up were complete and follow-up time was at least 24 months.

The exclusion criteria included: 1 ) spinal TB with acute pulmonary TB; 2 ) local kyphosis with Cobb angle beyond $30^{\circ} ; 3$ ) a history of lumbar spine surgeries by posterior approach.

\section{Preoperative preparation}

All patients received HRZE regimen for anti-TB treatment (isoniazid $300 \mathrm{mg}$, rifampicin $450 \mathrm{mg}$, ethambutol $1500 \mathrm{mg}$ and pyrazinamide $750 \mathrm{mg}$ ) at least 3 weeks before surgery. X-ray, CT and MRI were taken in all patients before operation to verify the damage of vertebrae and intervertebral disc and the compression of spinal canal. Surgery was performed when the patients restored appetite without low fever or night sweat, and anemia was improved as hemoglobin $\geq 100 \mathrm{~g} / \mathrm{L}$. However, if the patients showed 
a new onset of neurological symptoms or deterioration of primary neurological function, the surgery was performed immediately.

\section{Operative procedure}

Patients were placed in prone position after general endotracheal anesthesia, and a longitudinal midline incision was made. Then the musculus sacrospinalis was stripped off from the lamina; while the facet joints at contralateral side were exposed through Wiltse approach for instrumentation. Pedicle screws were placed into one to two normal vertebrae superior and inferior the pathological vertebrae. One rod was temporarily fixed at the none-debridement side to maintain the spinal stability. Then a unilateral laminectomy was performed to remove the caseous necrotic tissue, pus, granulation tissue, sequestrum and necrotic disc. The paravertebral or psoas abscess was drained off using a soft catheter tube. Another $3 \mathrm{~cm}$ long incision along the posterior superior iliac spine was made to expose the iliac crest and the cancellous bone was harvested for bone grafting (Fig.1); while at the lower lumbar spine the process of bone harvesting was done in the same incision. After flushing by saline, $450 \mathrm{mg}$ of isoniazid and $1.0 \mathrm{~g}$ of streptomycin powder were placed at the lesion site for local anti-tuberculosis treatment.

The following criteria should be satisfied when choosing nonstructural bone grafts: (1) bone defect $\otimes 1 / 3$ height of the vertebral body; (2) pedicle was intact at one side to ensure placing a pedicel screw. In addition, if newly formed bone bridged the lesion vertebra, nonstructural bone grafting is also chosen. The autogenous bone particles were placed into the intervertebral space and packed tightly. Otherwise, structural bone graft was chosen that the pediculectomy was performed and a titanium mesh filled with autogenous bone granules was implanted. During the whole procedure the spinous process and ligament complex were kept intact, and the neuro elements were protected meticulously (Fig.1). Finally, the rods were locked, and then the incision was closed. The pathological materials taken from the lesion site were sent for culture and histopathologic examination.

\section{Postoperative management}

Antibiotics were used to prevent infection in 3 days and the same regimen of anti-TB drugs as before surgery were prescribed to all patients and continued for 12 to 18 months. The drainage tube at the debridement side was kept for 7 days to inject with isoniazid $450 \mathrm{mg}$ and streptomycin $1.0 \mathrm{~g}$ every day for local anti-TB medication, whereas the drainage tube at contralateral side was removed within 48 hours. Blood routine test, ESR and CRP, liver and kidney function were periodically checked. A high protein diet was administered to patients during the whole therapeutic period. All patients were encouraged for mobilization under the protection of brace in 3 days postoperatively for quicker rehabilitation and the brace was worn for three to six months.

\section{Follow-up index and Statistical analysis}

The operation time, blood loss and duration of hospital stay were recorded. The visual analogue scores (VAS) of back pain and Oswestry disability index (ODI) were evaluated at the time points of pre-operation, 
1 week after operation, and last follow-up. X-ray films were taken regularly after operation and 1, 3, 6 and 12 months postoperatively and each year thereafter for each patient. The state of internal fixation and sagittal Cobb angle change were examined based on X-rays. While the computed tomography (CT) scan was examined at the first six-month postoperatively, and then taken periodically in every 6 months till intervertebral bone fusion was confirmed. Bone fusion was defined on thin-cut CT scan according to the standards as Burkus reported [16].

\section{Statistics}

Measurement data were recorded as mean $+/$ - standard deviation. The independent-sample $t$ test was used to compare the data between preoperative and postoperative measurements, and the data between two groups. While differences in bone fusion rate and number of complications between two groups were evaluated using Fisher exact test. Statistical significance was considered as $P$ value $₫ 0.05$.

\section{Results}

\section{Clinical outcomes}

Patients were divided into two groups due to the different methods of bone grafting. In group A, 27 patients received autogenous bone granules for grafting while 22 patients were treated with titanium mesh cage filled with autogenous bone in group $B$ after the similar procedure of posterior debridement and instrumentation. In group A, there were 16 males and 11 females, aged from 21 to 71 years old; while there were 12 males and 10 females in group B, aged from 26 to 69 years old. In group A, 8 patients were complicated with psoas major or iliac fossa abscess, while psoas major or iliac fossa abscess presented in 7 patients in group $B$.

Patients in both groups were followed up for at least 48 months. No significant difference of preoperative clinical data was found between the two groups. As shown in Table 1, the operation duration in group $A$ significantly shorter than that of group $B(4.1 \pm 0.7$ hours vs. $4.8 \pm 0.8$ hours, $P=0.000)$. The blood loss in group A was statistically less than that of group B ( $335 \pm 75 \mathrm{ml}$ vs. $462 \pm 120 \mathrm{ml}, \mathrm{P}=0.000)$. The VAS scores and ODI index did not present a significant difference between the two groups before surgery and improved significantly after surgery and at last follow-up in both groups, and no difference was observed between the two groups. After surgery, a significant improvement of ESR and CRP was found at the final follow-up, and no statistical difference was found between the two groups $(P=0.382$ and 0.058 respectively).

\section{Radiological evaluation}

As the films shown in Fig 2 and Fig 3, the TB lesions were cured in all patients, but different intervertebral bone fusion rate was demonstrated based on CT scan between the two groups. As shown in Table 2, the bone fusion rate at 6 -month postoperatively was $4 / 27$ in group $A$ and $7 / 22$ in group $B$, with no statistical difference $(P=0.185)$. While at 12 -month postoperatively and last follow-up, the fusion rate was 
significantly lower in the nonstructural group. The preoperative kyphotic Cobb angle was significantly improved after surgery in both groups. Despite a slight loss of Cobb angle was present at the last followup, no statistical difference was found between the two groups $(P=0.190)$. The length of fixation between the two groups, either one-segment or two-segment below and above the pathologic vertebrae, has no statistical difference $\mathbb{p}=0.586 \rrbracket$.

\section{Complications}

The incidence of complications between the two groups was not significantly different $(P=0.685)$. In group $A$, the TB recurred in one case with internal fixation loosening and kyphotic deformation of the lumbar spine as the patient didn't regularly take the drugs. After restart of anti-TB medication and external fixation with a rigid brace, the disease was cured, and intervertebral bony fusion was achieved with local kyphosis left. Cold abscess recurred in 2 cases but disappeared after CT guided puncture for abscess drainage. No implant failures were found, or no additional surgeries were undergone in the cases of incomplete grafted bone union during the follow-up.

In group B, cerebrospinal fluid leakage occurred in 2 cases, which were healed via periodically clipping drainage tube for 1 week. One case complained weakness and neuralgia of unilateral lower extremity after awaking from general anesthesia and recovered after the treatment with medication in three months. The wound nonunion happened in 1 case and was healed after dress change lasting for one month.

\section{Discussion}

This study compared the clinical effect of nonstructural autograft with titanium mesh filled up with autograft for bone graft and fusion in combination with posterior debridement and internal fixation for lumbar TB. The results demonstrated that the nonstructural autograft was equivalent to titanium mesh with autogenous bone for spinal reconstruction but had the advantages of diminished operation time and surgical trauma, except for delayed grafted bone fusion. Compared with implanting a titanium mesh cage, it is unnecessary to resect the pedicle for implanting bone granules into the lumbar vertebral space, therefor decreased the surgical trauma and maintained the spinal stability. Moreover, implanting bone

granules into the intervertebral space is much easier than placing a titanium mesh cage during the posterior procedure, decreasing the risk of neurological elements injury. Furthermore, it will save health cost for patients when choosing nonstructural bone grafting as cutting down the expense of titanium mesh cage.

The objective of surgical treatment for STB is debridement, decompression, prevention or correction of deformity, reconstruction of stability with bone grafting and internal fixation[17]. To date, however, the surgical approach hasn't reached a consensus among the surgeons yet and has been evolving for decades. The anterior approach enables to direct removal of the TB lesions, but the risks of injuring great vessels are relatively large especially at lower lumbar spine [18]. Combined anterior and posterior surgery improves the effects of debridement and spinal reconstruction, but the surgical trauma is enlarged [19]. 
The single posterior surgery has become popular nowadays as the advantages of less surgical trauma, easier kyphosis correction, stable fixation and simple techniques [20-22].

The typical STB affects intervertebral disc and adjacent vertebral bodies, resulting in spinal instability and kyphosis. Based on the three-column theory of Denis [23] and load-sharing principles [24], anterior column reconstruction by a structural bone grafting was mandatory after removing the affected vertebral bones and intervertebral discs. Many types of bone grafts have been used, such as tricortical iliac bone, titanium mesh cage filled with bone granules, rib bone and so on [10,25-26]. The autogenous strut harvested from iliac was frequently used to repair bone defects due to good osteogenesis, bone induction, bone conductibility and biocompatibility [27]. However, harvesting a tricortical bone may increase the complications of donor site including chronic pain and infection [28]. Harvesting spongy bone granules can be achieved by a tiny skin incision as 2 centimeters long in present study and fenestration of the posterior iliac crest, avoiding position change during the procedure and decreasing the donor site morbidity.

Titanium mesh cage used in the study was filled with resected cancellous bone from vertebral arch or harvested from the iliac crest. Although several studies reported that titanium mesh cage exhibited an important potential in reliable spinal reconstruction, high bone fusion, sufficient sagittal profile maintenance and lower implant-related problems[29], placing a titanium mesh cage from posterior approach in the lumbar spine would increase the operation duration and risk of injuring nerve roots[15]. The operation time was longer, and the intraoperative blood loss was more in the structural bone grafting group in our study, as placing a titanium mesh cage required extensive bone removal during the operation, resulting in increased surgical time and intraoperative bleeding. However, thanks to application of the analgesic, VAS scores of back pains improved in both group although paravertebral muscle extensively stripped and posterior spinal structures sacrificed in the structural bone grafting group.

The most concern about nonstructural bone grafting is the failure of support and breakage of instrumentation as it seems to violate the rule of anterior column reconstruction. Nevertheless, several studies have reported the success of using nonstructural bone graft in lumbar STB [13-15]. Our previous study also demonstrated the nonstructural bone graft had comparable bony fusion rate and even shorter fusion time compared with titanium mesh cage in single segment thoracic tuberculosis[12]. In the present study, however, the cancellous bone granules didn't exhibit the similar bone graft fusion time compared with titanium mesh cage filled with autogenous bone. We think the reason is insufficient volume of bone graft and resorption of grafted cancellous bone granules in the early stage. Owing to the preservation of the posterior structure and reliable pedicle screws fixation, however, complications like internal fixation failure or TB recurrence were not present in nonstructural bone graft group except one case who didn't regularly take the anti-TB drugs.

In the study, we found loss of Cobb angle was present in both groups. However, the Cobb angle loss in both groups is slight and the lumbar curvature are well maintained from post-operation to last follow-up, with no significant difference. However, the Cobb angle correction of nonstructural bone group was 
smaller than that of structural bone group, as the loose structure of granular bone could not bear the same supporting force as titanium mesh cage. To correct kyphosis through placement of contour rods and distracting intervertebral space, anterior support is necessary. In this case, structural bone grafts such as titanium mesh should be chosen instead of nonstructural bone grafts. Therefore, the essentials for success of nonstructural bone grafting are: 1) the posterior spinous process and ligamental complex should be retained during the operation to preserve the local stability. 2) during the procedure of debridement, the sclerotic bone and bone bridge between the anterior edge of vertebral bodies is preferred to be preserved. As more bony structure of vertebrae reserved, the volume of bone grafting needed is decreased and the demand for structural bone reconstruction could be avoided. 3) a rigid pedicle screw fixation should be guaranteed, without distraction of the vertebral interspace.

To the best of our knowledge, this is the first study comparing nonstructural bone with structural bone grafting in combination with posterior debridement and instrumentation for lumbar STB, and the results from follow-up time are good. However, the study still has some limitations. Firstly, this is a retrospective study of a small-scale of patients from a single center of institution. Second, the patients are not chosen randomly, so the selection bias is inevitable.

\section{Conclusions}

In conclusion, nonstructural bone grafting has less surgical trauma, simpler surgical procedure, and less intraoperative hemorrhage than structural bone grafting in the treatment of single segment lumbar STB after one stage posterior debridement and pedicle screws fixation. The nonstructural bone grafting is better suitable for the cases with smaller bony defect after debridement and slight local kyphosis without the need for correction.

\section{Abbreviations}

TB

tuberculosis

STB

spinal tuberculosis

\section{ERAS}

enhanced rehabilitation after surgery

ESR

erythrocyte sedimentation rate

CRP

C-reactive protein

CT

computed tomography

VAS

visual analogue scores 
ODI

Oswestry disability index

\section{Declarations}

This study was approved by the Institutional Review Board of the First Affiliated Hospital of Chongqing Medical University and the informed consent was obtained from all individual participants included in the study. Serial number of the project was 2019 Ke Yan Lun Li (No. 2019-123).

Not applicable of Consent for publication.

All data generated or analyzed during this study are included in this published article and its supplementary information files.

The authors declare that they have no competing interests.

The authors declare that the research didn't receive any source of funding.

Zenghui Zhao collected and interpreted the patient data and was a major contributor in writing the manuscript. Jianxiao Li analyzed the data and made the statistical analysis. Yong Zhu, Wei Luo and Xing Du made the patients follow-up work and analyzed the radiological films of the patients. Yunsheng Ou devised the research protocol and performed the operations.

All authors read and approved the final manuscript.

Not applicable of acknowledgements.

\section{References}

1. Not Dye C, Scheele S, Dolin P, Pathania V, Raviglione MC. Consensus statement. Global burden of tuberculosis: estimated incidence, prevalence, and mortality by country. WHO Global Surveillance and Monitoring Project. JAMA. 1999;282:677-86.

2. Jain AK, Kumar J. Tuberculosis of spine: neurological deficit. Eur Spine J. 2013;22:624-33.

3. Zeng H, Zhang P, Shen $X$, et al. One-stage posterior-only approach in surgical treatment of singlesegment thoracic spinal tuberculosis with neurological deficits in adults: a retrospective study of 34 cases. BMC Musculoskeletal Disorders. 2015;16:186.

4. Lee JS, Moon KP, Kim SJ and Suh KT. Posterior lumbar interbody fusion and posterior instrumentation in the surgical management of lumbar tuberculous spondylitis. J Bone Joint Surg Br. 2001;89-B:210-4.

5. Zhang HQ, Lin MZ, Shen KY, Ge L, Li JS, Tang MX, et al. Surgical management for multilevel noncontiguous thoracic spinal tuberculosis by single-stage posterior transforaminal thoracic 
debridement, limited decompression, interbody fusion, and posterior instrumentation (modified TTIF). Arch Orthop Trauma Surg. 2012;132:751-7.

6. Hassan K, Elmorshidy E. Anterior versus posterior approach in surgical treatment of tuberculous spondylodiscitis of thoracic and lumbar spine. Eur Spine J. 2016;25:1056-63.

7. Wang LJ, Zhang HQ, Tang MX, Gao QL, Zhou ZH, Yin XH. Comparison of Three Surgical Approaches for Thoracic Spinal Tuberculosis in Adult: Minimum 5-Year Follow-Up. Spine. 2017;42:808-17.

8. Güven O, Kumano K, Yalçin S, Karahan M, Tsuji S. A single stage posterior approach and rigid fixation for preventing Kyphosis in the treatment of spinal tuberculosis. Spine. 1994;199:1039-43.

9. Kumar MN, Joseph B, Manur R. Isolated posterior instrumentation for selected cases of thoracolumbar spinal tuberculosis without anterior instrumentation and without anterior or posterior bone grafting. Eur Spine J. 2013;22:624-32.

10. Qian J, Rijiepu A, Zhu B,Tian D, Chen L, Jing J. Outcomes of radical debridement versus no debridement for the treatment of thoracic and lumbar spinal tuberculosis. Int Orthop. 2016;40(10):2081-8.

11. Gao Y, Ou Y, Deng Q, He B, Du X, Li J. Comparison between titanium mesh and autogenous iliac bone graft to restore vertebral height through posterior approach for the treatment of thoracic and lumbar spinal tuberculosis. PLoS ONE. 2017;12:e0175567. https://doi.org/10.1371/journal.pone. 0175567.

12. Du X, Ou Y, Zhu Y, Zhao Z, Luo W, et al. One stage posterior debridement, non-structural bone graft in the surgical treatment of single segment thoracic tuberculosis: A retrospective single-center cohort study. International Journal of Surgery. 2019;65:134-9.

13. Xu Z, Wang X, Wu P, et al. Surgical treatment for mono-segmental lumbar tuberculosis by singlestage posterior debridement, compact bone grafting and posterior single-segment? Injury. 2015;46:1311-6.

14. Liu JM, Zhou Y, Peng AF, et al. One-stage posterior surgical management of lumbosacral spinal tuberculosis with non-structural autograft. Clin Neurol Neurosurg. 2017;153:67-72.

15. Liu JM, Chen XY, Zhou Y, et al. Is non-structural bone graft useful in surgical treatment of lumbar spinal tuberculosis? a retrospective case-control study. Medicine(Baltim.). 2016;95:e4677.

16. Burkus JK, Foley K, Haid RW, LeHuec JC. Surgical Interbody Research Group-radiographic assessment of interbody fusion devices: fusion criteria for anterior lumbar interbody surgery. Neurosurg Focus. 2001;10: E11.

17. Moon. Tuberculosis of the spine. Controversies and a new challenge. Spine. 1997;22:1791-7.

18. Benli IT, Kaya A, Acaroglu E. Anterior instrumentation in tuberculous spondylitis: is it effective and safe? Clin Orthop Relat Res. 2007;460:108-16.

19. Yang P, Zang Q, Kang J, et al. Comparison of clinical efficacy and safety among three surgical approaches for the treatment of spinal tuberculosis: a meta-analysis. Eur Spine J. 2016;25:3862-74.

20. Tang MX, Zhang HQ, Wang YX, Guo CF, Liu JY. Treatment of spinal tuberculosis by debridement, interbody fusion and internal fixation via posterior approach only. Orthop Surg. 2016;8:89-93. 
21. Rawall S, Mohan K, Nene A. Posterior approach in thoracolumbar tuberculosis: A clinical and radiological review of 67 operated cases. Musculoskelet Surg. 2013;97:67-75.

22. Fisahn C, Alonso F, Hasan GA, Tubbs RS, Dettori JR, Schildhauer TA, et al. Trends in spinal surgery for Pott's disease (2000-2016): an overview and bibliometric study. Global Spine J. 2017;7:821-8.

23. Denis $F$. The three-column spine and its significance in the classification of acute thoracolumbar spinal injuries. Spine. 1983;8:817-31.

24. Mccormack T, Karaikovic E, Gaines RW. The load sharing classification of spine fractures. Spine. 1994;19:1741-4.

25. Ozdemir HM, Us AK, Oğu"n T. The role of anterior spinal instrumentation and allograft fibula for the treatment of pott disease. Spine. 2003;28:474-9.

26. Christodoulou AG, Givissis P, Karataglis D, Symeonidis PD, Pournaras J. Treatment of tuberculos pondylitis with anterior stabilization and titanium cage. Clin Orthop Relat Res. 2006;444:60-5.

27. Sundararaj GD, Amritanand R, Venkatesh $K$, Arockiaraj J. The use of titanium mesh cages in the reconstruction of anterior column defects in active spinal infections: can we rest the crest? Asian Spine J. 2011;5:155-61.

28. Arrington ED, Smith WJ, Chambers HG, Bucknell AL, Davino NA. Complications of iliac crest bone graft harvesting. Clin Orthop Relat Res. 1996;329:300-9.

29. Zhang H, Zeng K, Yin X, Huang J, Tang M, Guo C. Debridement, internal fixation, and reconstruction using titanium mesh for the surgical treatment of thoracic and lumbar spinal tuberculosis via a posterior only approach: a 4-year follow-up of 28 patients. J Orthop Surg Res. 2015;10:150.

\section{Table}

Tab.1 Comparison of clinical and radiological data between two groups 


\begin{tabular}{|c|c|c|c|}
\hline \multirow[t]{2}{*}{ Mesurements } & Group A (27) & Group B (22) & P Value \\
\hline & Group A & Group B & P Value \\
\hline Sex囚male / female $\rrbracket$ & $16 / 11$ & $12 / 10$ & 0.746 \\
\hline Age $₫ X \pm S$, year $\rrbracket$ & $52.6 \pm 13.7$ & $49.5 \pm 10.5$ & 0.387 \\
\hline Operation duration(min) & $4.1 \pm 0.7$ & $4.8 \pm 0.8$ & 0.000 \\
\hline Blood loss (ml) & $335 \pm 75$ & $462 \pm 120$ & 0.000 \\
\hline Hospital stay & $14.3 \pm 4.5$ & $14.6 \pm 4.4$ & 0.841 \\
\hline
\end{tabular}

\begin{tabular}{llll}
\hline Preoperative & $3.8 \pm 1.2$ & $3.9 \pm 1.4$ & 0.895 \\
& & & \\
\hline Postoperative & $2.5 \pm 0.9$ & $2.2 \pm 0.9$ & 0.334 \\
\hline Last follow-up & $0.9 \pm 0.8$ & $1.1 \pm 0.9$ & 0.522 \\
\hline P value & 0.000 & 0.000 & \\
\hline ODI\&\% & & & \\
\hline Preoperative & $38.1 \pm 11.9$ & $41.2 \pm 14.0$ & 0.405
\end{tabular}

\begin{tabular}{clll}
\hline Postoperative & $31.6 \pm 7.8$ & $31.5 \pm 8.3$ & 0.953 \\
\hline Last follow-up & $15.0 \pm 5.2$ & $16.7 \pm 6.6$ & 0.347 \\
\hline P value* $^{*}$ & 0.000 & 0.000 & \\
\hline
\end{tabular}

$\operatorname{ESR}(\mathrm{mm} / \mathrm{h})$

$\begin{array}{llll}\text { Preoperative } & 58.4 \pm 17.4 & 59.1 \pm 19.6 & 0.891\end{array}$

\begin{tabular}{clll}
\hline Postoperative & $41.4 \pm 15.4$ & $41.6 \pm 13.3$ & 0.949 \\
\hline Last follow-up & $14.6 \pm 6.7$ & $16.3 \pm 6.6$ & 0.382 \\
\hline P value $^{\star}$ & 0.000 & 0.000 & \\
\hline CRP $(\mathrm{mg} / \mathrm{L})$ & & & \\
\hline Preoperative & $30.5 \pm 11.4$ & $32.5 \pm 16.5$ & 0.619
\end{tabular}




\begin{tabular}{clll} 
Postoperative & $17.9 \pm 7.3$ & $20.8 \pm 8.7$ & 0.211 \\
\hline Last follow-up & $6.2 \pm 2.9$ & $8.3 \pm 4.2$ & 0.058 \\
\hline P value* $^{*}$ & 0.000 & 0.000 & \\
\hline
\end{tabular}

Bone fusion rate

\begin{tabular}{llll}
\hline 6-month & $4 / 27$ & $7 / 22$ & 0.185 \\
\hline 12-month & $12 / 27$ & $20 / 22$ & 0.001 \\
& & & \\
\hline Last follow-up & $20 / 27$ & $22 / 22$ & 0.012 \\
\hline Cobb angle & & & \\
\hline
\end{tabular}

$\begin{array}{llll}\text { Preoperative } & 5.2 \pm 8.9 & 6.2 \pm 9.3 & 0.690\end{array}$

\begin{tabular}{llll}
\hline Postoperative & $-4.0 \pm 5.3$ & $-6.6 \pm 6.1$ & 0.062 \\
\hline Last follow-up & $-3.6 \pm 4.5$ & $-6.1 \pm 3.3$ & 0.032 \\
\hline P value $^{\star}$ & 0.807 & 0.000 & \\
\hline${\text { Cobb angle loss } \mathbb{\Xi}^{\circ} \rrbracket}^{\text {Complications }}$ & $1.3 \pm 1.9$ & $0.6 \pm 1.1$ & 0.153 \\
\hline 0 & $3 / 27$ & $4 / 22$ & 0.685
\end{tabular}

*measurements between the preoperative postoperative and last follow-up

\section{Figures}




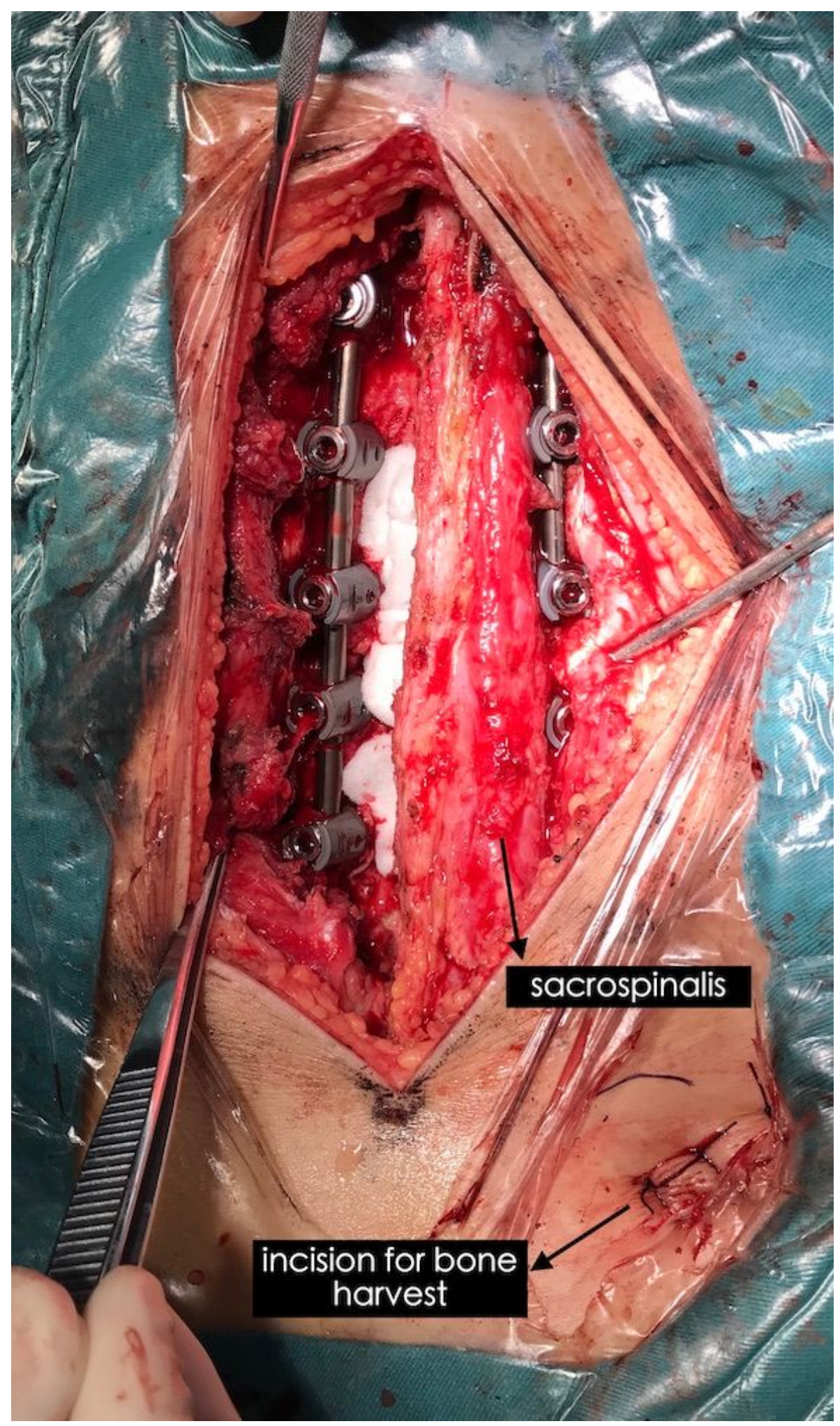

\section{Figure 1}

The picture taken during the operation shows the incision for exposing posterior structure and for iliac bone harvesting. 

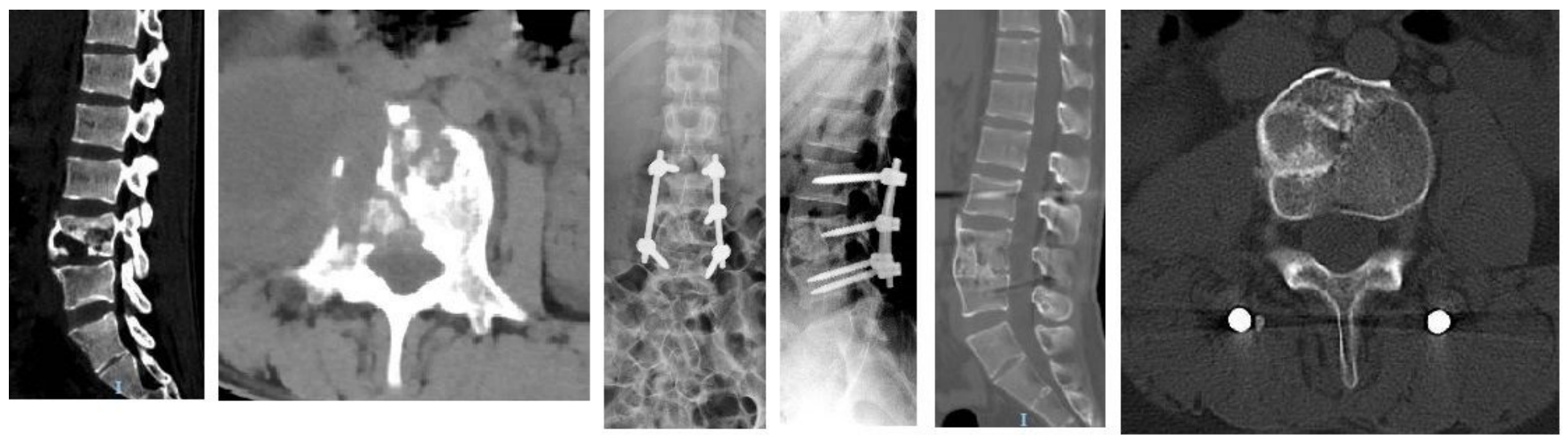

Figure 2

A 25 years old male with L4-5 TB reconstructed by none-structural bone a, b. Pre-OP sagittal and axial CT films demonstrate the destructed vertebral body and the iliac fossa abscess. $c$, $d$. The immediate post-OP X-ray films shows the internal fixation. e, f. Post-OP CT scan films demonstrate solid intervertebral bone fusion at 24 months' follow-up.
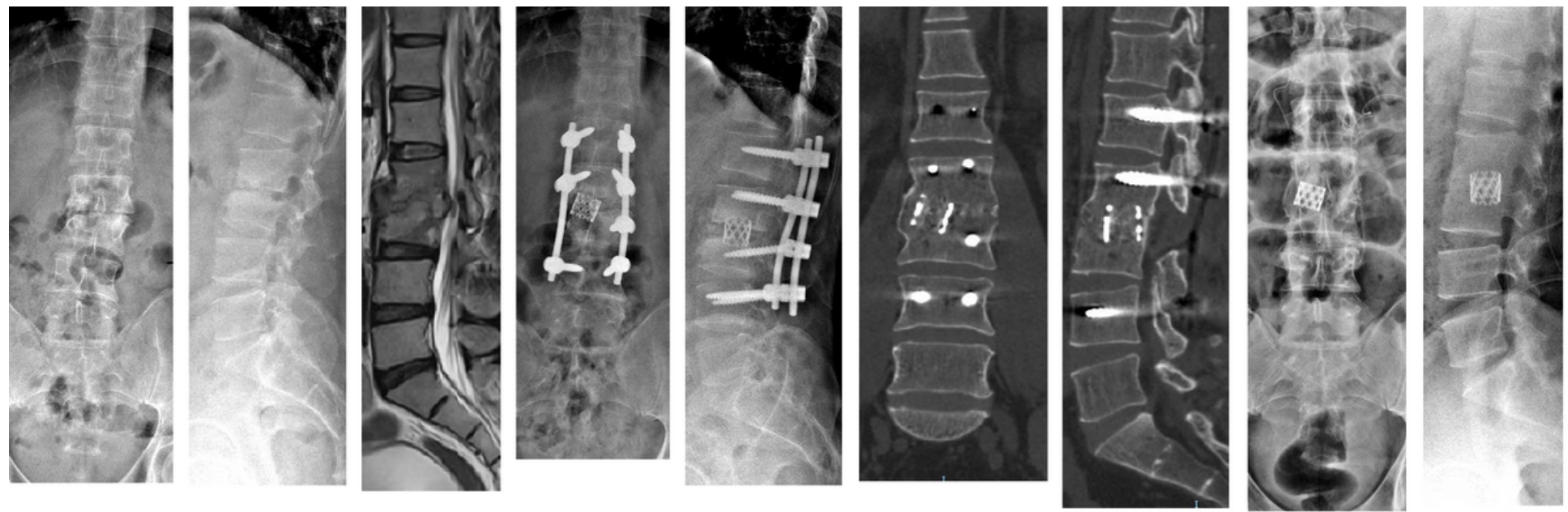

Figure 3

A 39 years old male presented with L2-3 TB reconstructed by titanium mesh cage. a, b, c. Pre-OP X-ray and MRI films show the vertebral and disc destruction of the spine and epidural abscess; $d$, e. The immediate post-OP X-ray films showed pedicle screws fixation with anterior spine reconstruction using titanium mesh cage. f, g. The CT films at 24-month show solid intervertebral bone fusion. $\mathrm{h}$, i. The X-ray films at 24-month post-OP show good spinal alignment after removing the instrumentation. 